\title{
CONGENITAL HEART DISEASE
}

\section{Influence of the pulmonary annulus diameter on pulmonary regurgitation and right ventricular pressure load after repair of tetralogy of Fallot}

\author{
A Uebing, G Fischer, M Bethge, J Scheewe, F Schmiel, J Stieh, J Brossmann, H H Kramer
}

Heart 2002;88:510-514

See end of article for authors' affiliations

Correspondence to: Dr A Uebing, Departmen of Paediatric Cardiology, Christian-Albrechts University of Kiel,

Schwanenweg 20, 24105 Kiel, Germany; vebing@pedcard.uni-kiel.de

Accepted 3 April 2002

\begin{abstract}
Objective: To assess the influence of the pulmonary annulus diameter after reconstruction of the right ventricular (RV) oufflow tract at repair of tetralogy of Fallot on pulmonary regurgitation and RV pressure load; and to evaluate the impact of pulmonary regurgitation on RV size and function.

Setting: Paediatric cardiology and diagnostic radiology departments of a tertiary referral centre. Patients: 67 patients were examined at a median of 4.8 years after repair of tetralogy of Fallot by means of biplane angiocardiography and magnetic resonance imaging (MRI).

Main outcome measures: Pulmonary annulus diameter and area, pulmonary regurgitant fraction, RV to left ventricular (LV) systolic pressure ratio, RV end diastolic volume, and RV ejection fraction were assessed.

Results: There was a significant positive correlation between pulmonary annulus area indexed to body surface area and pulmonary regurgitation (angiocardiography: $r=0.55, p<0.001 ; M R I: r=0.59$, $p<0.001$ ). No significant correlation was found between pulmonary annulus index and RV to LV systolic pressure ratio even in patients with small pulmonary annulus areas $(r=-0.24, \mathrm{NS})$. Pulmonary regurgitant fraction was positively correlated with normalised RV end diastolic volume (angiocardiography: $r=0.42, p<0.05 ;$ MRI: $r=0.56, p<0.01$ ). RV ejection fraction decreased with increasing pulmonary regurgitation (angiocardiography: $r=-0.42, p<0.05$; MRI: $r=-0.41, p<0.05$ ).

Conclusions: The extent of pulmonary regurgitation after tetralogy of Fallot repair correlates with the postoperative size of the pulmonary annulus and is closely correlated with the enlargement of the RV. An enlargement of the pulmonary annulus to the second lower standard deviation of normal results in a decrease of pulmonary regurgitation and is sufficient to achieve adequate $\mathrm{RV}$ pressure unloading.
\end{abstract}

7 etralogy of Fallot can be surgically repaired nowadays even in young infants with low early mortality. ${ }^{1}$ However, in some series the need for transannular patch enlargement of the right ventricular (RV) outflow tract has increased up to $88 \% .^{12}$ The main concern with using a transannular patch enlargement is that a wide enlargement of the pulmonary annulus may aggravate pulmonary regurgitation, which has deleterious effects on long term outcome. Pulmonary regurgitation reduces exercise capacity ${ }^{3}$ and is associated with RV enlargement, ${ }^{4}$ late ventricular arrhythmias, and sudden cardiac death. ${ }^{5}$ The present retrospective study assessed the influence of the pulmonary annulus diameter (PAD) after surgical RV outflow tract reconstruction on pulmonary regurgitation and RV pressure load. Additionally, the impact of pulmonary regurgitation on RV size and function was analysed.

\section{PATIENTS AND METHODS}

The study group consisted of 67 patients (39 male and 28 female patients) who underwent repair of tetralogy of Fallot at a median age of 1.8 (range $0.2-11.2$ ) years at the University Hospital of Kiel, Germany. These patients were taken from a total number of 236 who underwent repair between 1975 and 1998 and were selected on the basis that quantitative data of $\mathrm{RV}$ volumes and pulmonary insufficiency were available from either angiocardiography or magnetic resonance imaging (MRI). Patients with double outlet RV, pulmonary atresia, or absent pulmonary valve were not considered. In 10 patients a primary palliative procedure was performed in the neonatal period (six balloon dilatation of the pulmonary valve, three modified Blalock-Taussig-shunt, and one Waterston-Cooleyshunt).
Outflow tract repair was performed in three ways:

- transannular patch of the RV outflow tract $(n=50)$

- muscular resection, subvalvar outflow tract patch, and pulmonary valvotomy $(\mathrm{n}=11)$

- muscular resection and pulmonary valvotomy $(\mathrm{n}=6)$.

Patients with any residual intracardiac shunting $(n=1)$ or significant peripheral pulmonary stenosis (invasive systolic pressure gradient $\geqslant 25 \mathrm{~mm} \mathrm{Hg} ; \mathrm{n}=11$ ) were excluded from the study. The parents of all patients gave their informed consent for the examinations.

\section{Cardiac catheterisation}

In all patients diagnostic cardiac catheterisation was performed at a median of 4.8 (range 0.1-22.6) years after surgery. Blood samples for oximetry were taken before the first angiocardiogram was recorded. Pressures were recorded in the left, right, and main pulmonary artery and in the right and left ventricle using a side hole catheter. Zero pressure was referenced to the level of the right atrium in the lateral projection. If left ventricular pressure was not measured, then noninvasive arterial blood pressures were taken and systolic arterial pressure was used for further calculations.

Abbreviations: BSA, body surface area; FLASH, fast low angle shot; $M R I$, magnetic resonance imaging; PAD, pulmonary annulus diameter; $\mathrm{PAI}$, pulmonary annulus index; $\mathrm{PRF}$, pulmonary regurgitant fraction; RV, right ventricle; RVEDV, right ventricular end diastolic volume, RVEF, right ventricular ejection fraction; RVESV, right ventricular end systolic volume 
Angiocardiography

In all patients a biplane (posteroanterior and lateral) RV angiocardiogram was recorded after injection of contrast medium $(0.5-1.5 \mathrm{ml} / \mathrm{kg})$ at $50 \mathrm{frames} / \mathrm{s}$. The angiocardiograms were recorded on magnetic tape or automatically digitised for later analysis.

\section{Pulmonary annulus diameter}

The diameter of the RV-pulmonary trunk junction at the level of the preoperative pulmonary annulus was measured from the lateral angiogram. ${ }^{6}$ In this study the term "pulmonary annulus diameter" was used also for the postoperative status. In one patient reliable measurement of the PAD was impossible because of low contrast.

The pulmonary annulus index (PAI) was calculated according to the following formula:

$$
\pi \times \frac{\left(\frac{\mathrm{PAD}[\mathrm{mm}]}{2}\right)^{2}}{\mathrm{BSA}\left[\mathrm{m}^{2}\right]}=\mathrm{PAI}
$$

where BSA is body surface area.

\section{RV volume determination}

In 47 patients RV end diastolic (RVEDV) and end systolic volumes (RVESV) were calculated from biplane RV angiocardiograms using the area length method. All volumes were corrected with factors appropriate for projection and cardiac phase. The method has previously been described in detail. ${ }^{7}$

$\mathrm{RV}$ ejection fraction (RVEF) was calculated according to the following formula:

$$
\frac{\text { RVEDV }- \text { RVESV }}{\text { RVEDV }} \times 100=\operatorname{RVEF}[\%]
$$

RVEDV and RVESV were normalised for the corresponding BSA with an exponent of 1.219 since ventricular volumes increase in an overproportional manner with respect to BSA. ${ }^{7}$

\section{Angiocardiographic quantification of pulmonary} insufficiency

In 35 patients pulmonary insufficiency was quantified using $x$ ray densitometry. In 23 patients both $x$ ray densitometry and angiocardiographic volume determination were performed.

$x$ Ray densitometry is a dye dilution technique. After injection of contrast medium into the RV the total amount of this indicator was measured over several heart cycles within an electronic sampling window covering the silhouette of the RV in the lateral $x$ ray plane using densitometry. The ratio of changes in amplitudes of the resulting densitogram represents the reflux of contrast medium (difference of deflections between end diastole and preceding end systole) and the systolic forward flow (difference of deflections between end diastole and following end systole). Thus, densitometry allows the calculation of the pulmonary regurgitant fraction (PRF), which is calculated as the ratio of regurgitant volume to stroke volume. A detailed description of the method was given by Bürsch and colleagues ${ }^{8}$ and Simon and associates.

\section{Magnetic resonance imaging}

In 34 patients cardiac MRI was performed on the day of cardiac catheterisation to determine RV volume parameters and PRF. In five patients the determination of RV volume (two) or PRF (three) failed because of technical problems. Thus, a complete set of MRI data was obtained for 29 patients.

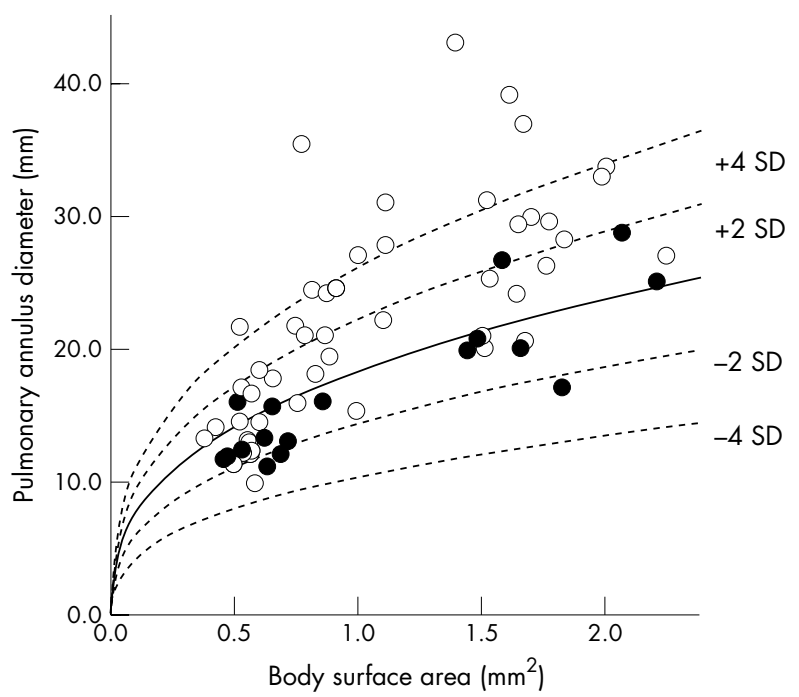

Figure 1 Postoperative pulmonary annulus diameter as measured by angiocardiography versus body surface area. Open circles: use of a transannular patch at repair; closed circles: no use of a transannular patch. The unbroken line is the regression line relating pulmonary annulus diameter and body surface area in children and adolescents without heart disease (broken line indicate \pm second and fourth standard deviations). ${ }^{6}$

The examinations were performed using a Vision system (Siemens, Erlangen, Germany) operating at 1.5 T. Conventional ECG triggered spin echo images were used to determine the position of the ventricles and the main pulmonary artery. All MRI data acquisition was triggered by the $\mathrm{R}$ wave of the ECG. MRIs were analysed using the ARGUS software implemented on the Siemens system.

$\mathrm{RV}$ volume determination by MRI

Transverse multisection multiphase gradient echo MRI of the ventricles was performed for quantification of RV volumes. The technique is described in detail elsewhere. ${ }^{10}{ }^{11} \mathrm{~A}$ transverse stack of multisection multiphase images (4-6 sections, 12-16 phase images per heart cycle) was acquired using a two dimensional fast low angle shot (FLASH) cine sequence (repetition time $40 \mathrm{~ms}$, echo time $6.8 \mathrm{~ms}$, flip angle $30^{\circ}$, slice thickness $10 \mathrm{~mm}$, no interleaf, field of view $300 \times 300 \mathrm{~mm}$, matrix $256 \times 256$, acquisition time $4 \mathrm{~min} 44 \mathrm{~s}$ ). RVEDV and RVESV were measured from endocardial contours manually traced on the multisection multiphase gradient echo images. The volumes were calculated by adding the ventricular cavity areas multiplied by section thickness with correction for the interslice gap. Papillary muscles and moderator band were not added to the ventricular area. RVEDV and RVESV were normalised for the corresponding BSA to the power of 1.219 in analogy with the angiocardiographic volumes. For comparison with normal values the volumes were normalised for BSA without an exponent. This method was also used in the study where normal values were obtained. ${ }^{10}$

\section{Quantification of pulmonary insufficiency by MRI}

Pulmonary insufficiency was quantified using the magnetic resonance velocity mapping technique, which is a modified gradient echo sequence that uses a velocity encoding magnetic field gradient in the direction of flow. ${ }^{11}{ }^{12}$ The conventional spin echo images were used to select the oblique imaging plane for magnetic resonance velocity mapping of flow in the main pulmonary artery. The imaging plane was positioned halfway between the pulmonary annulus and the bifurcation of the main pulmonary artery perpendicular to the direction of flow. Through-plane velocities were quantified in a single slice by using a two dimensional FLASH velocity encoded 


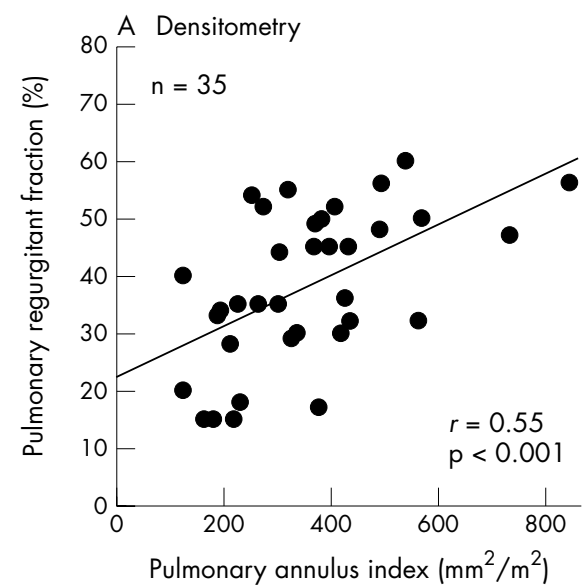

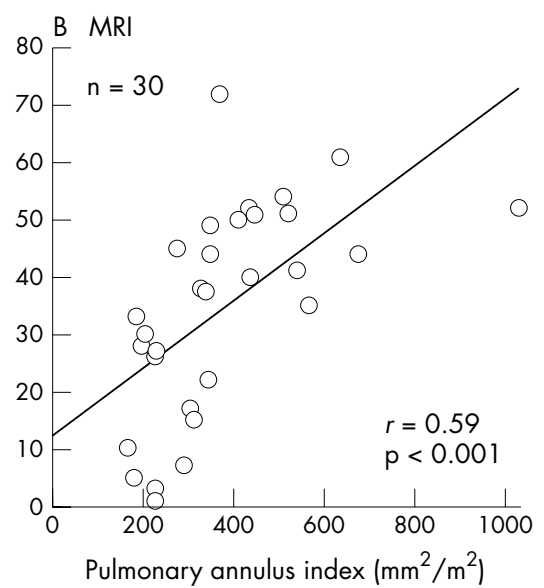

Figure 2 Pulmonary regurgitant fraction versus pulmonary annulus index. (A) Pulmonary regurgitant fraction measured by densitometry. (B) Pulmonary regurgitant fraction measured by magnetic resonance imaging (MRI). sequence (repetition time $24 \mathrm{~ms}$, echo time $5 \mathrm{~ms}$, flip angle $30^{\circ}$, slice thickness $6 \mathrm{~mm}$, field of view $320 \times 320 \mathrm{~mm}$, matrix $256 \times 256 \mathrm{~mm}$, pixel size $1.25 \times 1.25 \mathrm{~mm}$, acquisition time $1 \mathrm{~min} 56 \mathrm{~s}$ ). The cardiac cycle was divided into 20-30 time frames with temporal resolution ranging from $21-36 \mathrm{~ms}$ depending on the individual patient's heart rate. Volume of flow was calculated by tracing the region of interest along the inner borders of the pulmonary artery wall in each time frame of a velocity map series. For every time frame instantaneous volumetric flow was calculated by multiplying spatial average flow velocity with the area of the region of interest. Integration of all instantaneous volumetric flow data yielded total volume flow per cardiac cycle. PRF was calculated as the ratio of regurgitant flow to forward flow.

\section{Statistical analysis}

Correlation between haemodynamic, angiocardiographic, and MRI variables was tested by linear regression analysis by the least squares method. The patient group was compared with normal children by Student's $t$ test. All analyses were performed using Xact 7.06 statistical software (SCIlab GmbH, Hamburg, Germany). A probability value of $p<0.05$ was considered to indicate significance.

\section{RESULTS}

The postoperative PAD varied widely in the study patients (fig 1). Mainly older patients operated on in the 1970s and 1980s had greatly enlarged PADs, which are far above the upper second standard deviation of a normal mean value related to the BSA, ${ }^{6}$ while the majority of younger patients operated on more recently had normal PAD.

There was a significant positive correlation between PAI and pulmonary insufficiency as determined both by $x$ ray densitometry $(r=0.55, \mathrm{p}<0.001)$ and MRI $(r=0.59$, $\mathrm{p}<0.001$; fig 2) indicating that a wide extension of the pulmonary annulus aggravates pulmonary insufficiency.

No correlation was found between age at operation and PRF measured by $x$ ray densitometry $(r=0.02$, NS) or MRI $(r=0.20$, NS). Additionally, there was no significant relation between time of follow up and pulmonary insufficiency. Similar results were obtained with both methods (densitometry: $r=0.28$, NS; MRI: $r=0.23$, NS).

There was no correlation between RV to left ventricular systolic pressure ratio and PAI ( $r=-0.24$, NS; fig 3$)$. Even in patients with small pulmonary annulus indices close to the fourth lower standard deviation, RV pressure load was not significantly increased.

PRF was positively correlated with normalised RVEDV (fig $4)$. We obtained similar results by angiocardiographic or densitometric $(r=0.42, \mathrm{p}<0.05)$ and MRI analyses $(r=0.56$, $p<0.01$ ), which showed that the severity of pulmonary insufficiency determines the degree of the enlargement of the RV.

Normalised RVEDV was larger in our group of patients than in healthy children. However, the difference was significant only for angiocardiographic measurement (mean (SD) $86.0(26.3) \mathrm{ml} / \mathrm{m}^{2 \times 1.219}$ v $\left.73.1(11.8) \mathrm{ml} / \mathrm{m}^{2 \times 1.219}, \mathrm{p}<0.001\right),{ }^{7}$ not for MRI analysis $\left(77.7(28.1) \mathrm{ml} / \mathrm{m}^{2} v 70.0(9.0) \mathrm{ml} / \mathrm{m}^{2}\right){ }^{10}$ Furthermore, normalised RVESV was larger in our group of patients than the normal values. The difference was significant for angiocardiographic and for MRI measurements (RVESV measured by angiocardiography $v$ normal value $: 38.5$ (14.3) $\mathrm{ml} / \mathrm{m}^{2 \times 1.219} v 26.9$ (8.6) $\mathrm{ml} / \mathrm{m}^{2 \times 1.219}, \mathrm{p}<0.0001$; RVESV measured by MRI $v$ normal value ${ }^{10}: 36.9(15.6) \mathrm{ml} / \mathrm{m}^{2} v 21.0$ (5) $\mathrm{ml} / \mathrm{m}^{2}$ ).

We found a significant relation between age at operation and postoperative angiocardiographic RVEDV normalised to $\mathrm{BSA}^{1.219}$ and between time of follow up and angiocardiographic RVEDV normalised to $\mathrm{BSA}^{1.219}(r=0.45$ and $r=0.58$, respectively, both $\mathrm{p}<0.01)$. There was no relation between any of these variables when volumes were calculated by MRI.

An overall reduction of RVEF was observed in our patient group relative to normal values obtained from healthy children. For angiocardiographic and MRI volume determinations, the reduction was significant (RVEF measured by angiocardiography $v$ normal value ${ }^{7}: 55.5(6.1) \%$ v 63.3 (6.8)\%, $\mathrm{p}<0.05$; RVEF measured by MRI $v$ normal value ${ }^{10}: 53.2$ $(8.7) \% \vee 70(4) \%, \mathrm{p}<0.05)$.

Additionally, we found a negative correlation of $r=-0.4 \mathrm{l}$ $(\mathrm{p}<0.05)$ between RVEF and PRF determined by MRI and a

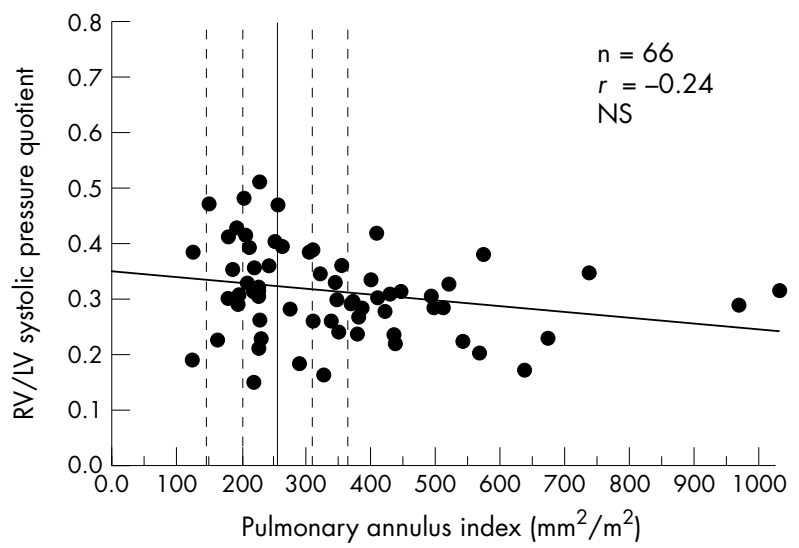

Figure 3 Right to left ventricular (RV/LV) systolic pressure quotient versus pulmonary annulus index. The unbroken vertical line indicates the mean value in children and adolescents without heart disease (broken lines indicate \pm second and fourth standard deviations). ${ }^{6}$ 

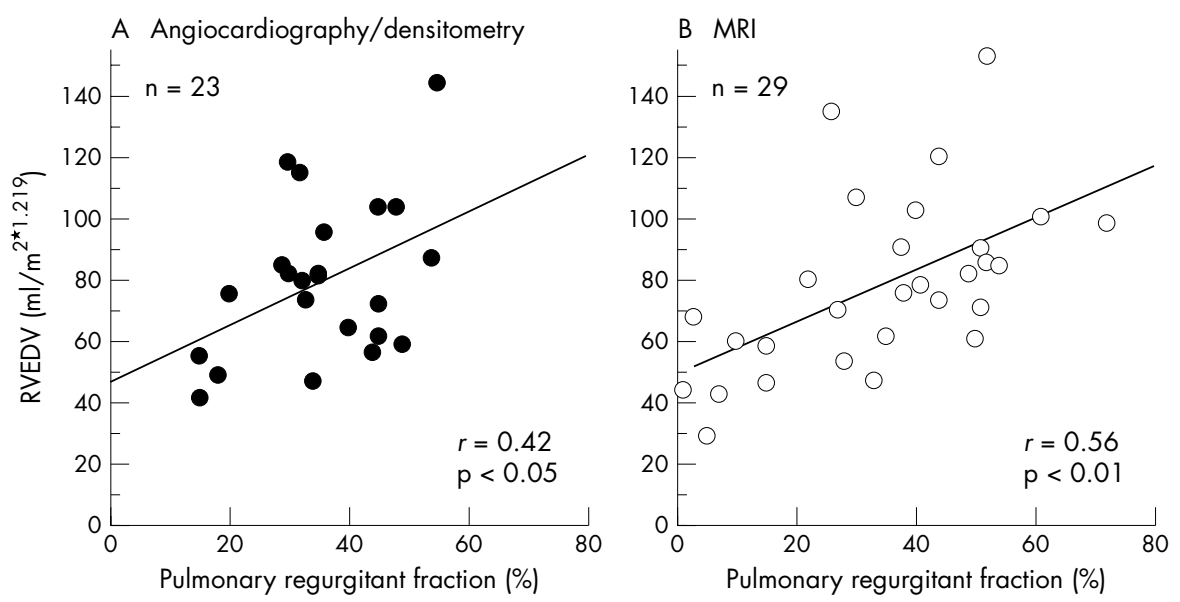

Figure 4 Right ventricular end diastolic volume (RVEDV) versus pulmonary regurgitant fraction. (A) RVEDV and pulmonary regurgitant fraction determined by angiocardiography or densitometry. (B) RVEDV and pulmonary regurgitant fraction determined by MRI.

similar correlation of $r=-0.42(\mathrm{p}<0.05)$ between these variables when ejection fraction was calculated by angiocardiography and PRF by $x$ ray densitometry (fig 5 ).

RVEF was negatively correlated with normalised RVESV regardless of which technique was used for volume determination (angiocardiography: $r=-0.54, \quad \mathrm{p}<0.001$; MRI: $r=-0.56, \mathrm{p}<0.01)$.

RVEF was not correlated with age at operation. Similar results were obtained by angiocardiographic $(r=-0.02$, NS) and MRI analyses ( $r=-0.28$, NS). In addition, we did not find a relation between RVEF and time of follow up regardless of which technique was used for calculation of RVEF (angiocardiography: $r=0.02$, NS; MRI: $r=-0.21$, NS). These results show that RVEF is not affected by variability of age at operation and time of follow up in our study group.

\section{Comparison between angiocardiography or densitometry and MRI}

In 12 patients PRF was calculated by $x$ ray densitometry and MRI. The correlation between the methods was significant $(r=0.75)$. The regression equation was determined to be $\operatorname{PRF}($ densitometry $)=0.93 \operatorname{PRF}(\mathrm{MRI})+5.0(\mathrm{p}<0.01)$.

In 23 patients RVEDV was calculated by angiocardiography and MRI, and we found a highly significant correlation for RVEDV measured by both methods ( $r=0.89$; regression equation: RVEDV(angiography) $=1.2 \operatorname{RVEDV}(\mathrm{MRI})+8.1$; $\mathrm{p}<0.0001)$

\section{DISCUSSION}

The ideal purpose of RV outflow tract reconstruction at repair of tetralogy of Fallot is to achieve near normal RV pressures and to preserve pulmonary valve competence. The latter task is of great importance for long term outcome because pulmonary insufficiency reduces exercise capacity and is associated with late ventricular arrhythmias and sudden cardiac death. $^{35}$

In the present retrospective study we showed that the extent of pulmonary insufficiency correlates with the postoperative size of the pulmonary annulus. We found sufficient pressure unloading even in patients with very small PADs (between the second and fourth lower standard deviation). Therefore, our results show that even slighter extensions of the outflow tract, as was often performed previously, are sufficient to achieve adequate pressure unloading of the RV. Because of these findings in recent years our cardiac surgeons have been using a transannular patch only if the angiocardiographic PAD lies below the lower fourth standard deviation of the normal mean value. In cases where a transannular patch is used, the enlargement of the pulmonary annulus is nowadays restricted to diameters within the range of the lower second standard deviation-that is, the lowest normal value.

Not only the width of the pulmonary annulus may contribute to pulmonary insufficiency but also branch or distal pulmonary stenoses, which were excluded from the present analysis, or residual pulmonary valve tissue after repair. In the latter respect we analysed a heterogeneous group of patients. As we found a significant correlation between PAD and PRF, ignoring eventual residual valve tissue, an even closer correlation may be expected in a more uniform group of patients. However, it is not likely that residual pulmonary valve tissue can reduce the degree of pulmonary insufficiency in patients with enlarged PADs.
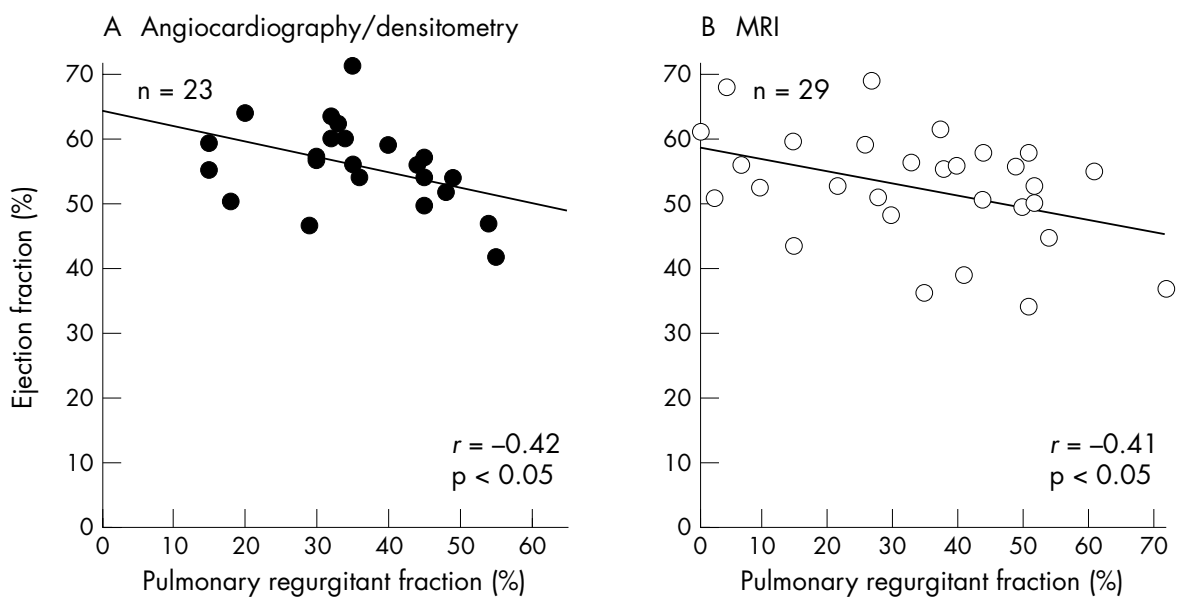

Figure 5 Right ventricular ejection fraction versus pulmonary regurgitant fraction. (A) Ejection fraction and pulmonary regurgitant fraction determined by angiocardiography or densitometry. (B) Ejection fraction and pulmonary regurgitant fraction determined by MRI. 
Our study confirms previous results obtained by angiocardiography and MRI, which showed a close correlation between postoperative pulmonary insufficiency and RV enlargement. ${ }^{43}$ However, in their angiographic study, Redington and colleagues ${ }^{4}$ found no correlation between either age at operation or time of follow up and RVEDV. This is in accordance with our MRI results but not with the respective angiocardiographic data. This discrepancy is explained by the fact that an overproportional number of patients operated on in early infancy were examined by angiocardiography less than five years after corrective surgery. In these patients the smaller RV is explained by the recent change in our surgical policy, which aims at reducing the frequency and extent of transannular patching. The correlation between angiocardiographic RVEDV and both age at operation and time of follow up reflects, at least in part, this change in surgical approach. In the group of patients examined by MRI we did not find an overproportional number of patients who were operated on in early infancy and had small RVs. This group of patients is evenly distributed by age at operation and follow up time and, therefore, does not reflect the change in surgical strategy.

In our study RV volumes were obtained on the basis of biplane angiocardiography and MRI. Volume from biplane angiocardiograms was calculated using the area length method. All volumes were corrected by factors appropriate for projection and cardiac phase as described by Lange and colleagues. $^{7}$ The correction factors were obtained from cast studies of RVs of normal shape. We used these factors in the present study for volume calculation of reconstructed, volume loaded and hence enlarged and abnormally shaped RVs.

RV volume calculation using MRI after repair of tetralogy of Fallot has been previously reported. ${ }^{11}{ }^{13}$ Volumetry by angiocardiography and MRI showed similar relations between PRF and RVEDV, as well as RVEF (figs 4 and 5). Additionally, we found a good correlation between RV volume as measured by angiocardiography and MRI. Therefore, despite the methodological uncertainties of angiocardiographic volumetry of abnormally shaped ventricles, there is a clinically sufficient accordance of both methods.

The degree of pulmonary insufficiency was quantified by $x$ ray densitometry. The accuracy of this technique has been verified by electromagnetic flow measurement in an animal model. ${ }^{9}$ A good correlation between PRF measured by densitometry and by MRI shows the good quality of both techniques.

We found an overall reduced ejection fraction in our group of patients who had undergone repair of tetralogy of Fallot in comparison with normal values for angiocardiographic and MRI analyses. With increasing volume load caused by pulmonary insufficiency, RVEF decreased. These observations are in accordance with other studies ${ }^{13}{ }^{14}$ but contrast in some ways with the findings of Redington and colleagues, ${ }^{4}$ who did not find a relation between PRF and RVEF in a group of 12 patients. In contrast to our study group only three of their patients had a PRF of more than $30 \%$ of stroke volume. It seems that PRF reduces ejection fraction only above a certain threshold.

Ejection fraction is calculated as the ratio of stroke volume to end diastolic volume. In our group of patients we found a reduction in RVEF and an increase in RVEDV as a consequence of volume load (fig 4). This reduction in ejection fraction may partly reflect the increase in RVEDV. Therefore, ejection fraction alone does not seem to be a distinct measure of myocardial performance of volume loaded enlarged ventricles. Progressive deterioration of myocardial function finally results in decreasing stroke volume and as a consequence in increasing RVESV. The end systolic volume should therefore additionally be taken into account in terms of estimating myocardial function of a volume loaded ventricle, as it has the advantage of reflecting both RV enlargement and myocardial function.

We performed a retrospective study and therefore analysed all available data on pulmonary insufficiency and RV volumes obtained by angiocardiography, densitometry, or MRI. No data were excluded from analysis. The lack of data in some patients is caused by the retrospective setting of the study. We tried to maximise homogeneity by excluding patients with double outlet RV, pulmonary atresia, or absent pulmonary valve. Nevertheless, in terms of age at operation, heterogeneity is unavoidable because the surgical policy did change over the past two decades towards primary repair in early infancy. One would expect an influence of age at operation and time of follow up on the amount of pulmonary insufficiency. As we did not find a relation between pulmonary insufficiency and one of these variables we can exclude an impact on the relation between the width of the pulmonary annulus and pulmonary insufficiency. In addition we can exclude an impact of age at operation and time of follow up on RVEF because we did not find any correlation between these variables. PAD was widely scattered in our patient group and hence the group seems to be suitable for that study. The presented data allow the conclusion that a wide enlargement of the pulmonary annulus at repair aggravates pulmonary insufficiency (fig 2 ) and is not necessary to achieve adequate pressure unloading (fig 3 ).

\section{Conclusions}

Pulmonary regurgitation after repair of tetralogy of Fallot correlates with the postoperative size of the pulmonary annulus and is a closely correlated with RV size. A slight extension of the pulmonary annulus to only the lower second standard deviation - that is, the lowest normal value-is recommended because it results in a decrease of pulmonary regurgitation and is sufficient to achieve adequate RV pressure unloading.

\section{ACKNOWLEDGEMENTS}

This work was presented in part T the XXXV annual general meeting of the Association for European Paediatric Cardiology, Strasbourg, 14-17 June 2000.

\section{REFERENCES}

1 Pigula FA, Khalil PN, Mayer JE, et al. Repair of tetralogy of Fallot in neonates and young infants. Circulation 1999;100(19 suppl):I1157-61.

2 Hirsch JC, Mosca RS, Bove EL. Complete repair of tetralogy of Fallot in the neonate: results in the modern Era. Ann Surg 2000;232:508-14.

3 Carvalho JS, Shinebourne EA, Busst C, et al. Exercise capacity after complete repair of tetralogy of Fallot: deleterious effects of residual pulmonary regurgitation. Br Heart J 1992;67:470-3.

4 Redington AN, Oldershaw PJ, Shinebourne EA, et al. A new technique for the assessment of pulmonary regurgitation and its application to the assessment of right ventricular function before and after repair of tetralogy of Fallot. Br Heart J 1988;60:57-65.

5 Marie PY, Marcon F, Brunotte F, et al. Right ventricular overload and induced sustained ventricular tachycardia in operatively "repaired" tetralogy of Fallot. Am J Cardiol 1992:69:785-9.

6 Sievers HH, Onnasch DG, Lange PE, et al. Dimensions of the great arteries, semilunar valve roots, and right ventricular oufflow tract during growth: normative angiocardiographic data. Pediatr Cardiol 1983;4:189-96.

7 Lange PE, Onnasch D, Farr FL, et al. Angiocardiographic right ventricular volume determination: accuracy, as determined from human casts, and clinical application. Eur J Cardiol 1978;8:477-501.

8 Bürsch JH, Heintzen PH, Simon R. Videodensitometric studies by a new method of quantitating the amount of contrast medium. Eur J Cardiol 1974:1:437-46.

9 Simon R, Callesen C, Heintzen PH. [Videodensitometric determination of the regurgitant fraction in pulmonic insufficiency by measuring the amount of indicator]. Basic Res Cardiol 1973;68:509-20.

10 Helbing WA, Rebergen SA, Maliepaard C, et al. Quantification of right ventricular function with magnetic resonance imaging in children with normal hearts and with congenital heart disease. Am Heart J $1995 ; 130: 828-37$

11 Rebergen SA, Chin JG, Ottenkamp J, et al. Pulmonary regurgitation in the late postoperative follow-up of tetralogy of Fallot: volumetric quantitation by nuclear magnetic resonance velocity mapping Circulation 1993;88:2257-66

12 Rebergen SA, van der Wall EE, Doornbos J, et al. Magnetic resonance measurement of velocity and flow: technique, validation, and cardiovascular applications. Am Heart J 1993;126:1439-56.

13 Helbing WA, Niezen RA, Le Cessie S, et al. Right ventricular diastolic function in children with pulmonary regurgitation after repair of tetralogy of Fallot: volumetric evaluation by magnetic resonance velocity mapping J Am Coll Cardiol 1996;28: 1827-35.

14 Lange PE, Onnasch DG, Bernhard A, et al. Left and right ventricular adaptation to right ventricular overload before and after surgical repair of tetralogy of Fallot. Am J Cardiol 1982;50:786-94. 